\title{
High sugar-sweetened beverage intake frequency is associated with smoking, irregular meal intake and higher serum uric acid in Taiwanese adolescents
}

\author{
Y. H. Shih ${ }^{1}$, H. Y. Chang ${ }^{1,2} *$ (D) H. C. Wu ${ }^{3}$, F. F Stanaway ${ }^{4}$ and W. H. Pan ${ }^{5}$ \\ ${ }^{1}$ Institute of Population Health Sciences, National Health Research Institutes, Miaoli, Taiwan \\ ${ }^{2}$ National Yang-Ming University, Taipei, Taiwan \\ ${ }^{3}$ Department of Food Nutrition, College of Human Science and Technology, Chung Hwa University of Medical Technology, Tainan, Taiwan \\ ${ }^{4}$ Sydney School of Public Health, The University of Sydney, Camperdown, NSW 2006, Australia \\ ${ }^{5}$ Institute of Biomedical Sciences, Academia Sinica, Taipei, Taiwan
}

(Received 26 July 2019 - Final revision received 14 January 2020 - Accepted 16 January 2020)

Journal of Nutritional Science (2020), vol. 9, e7, page 1 of 10

doi:10.1017/jns. 2020.2

\section{Abstract}

Types of sugar-sweetened beverages (SSB) can differ greatly between countries, with greater consumption of sweetened tea in Asia. This study aimed to understand changes in SSB consumption by adolescents in Taiwan over 18 years and their association with demographic characteristics and clinical outcome. This study used survey data from the 1993-1996 and 2010-2011 Nutrition and Health Surveys in Taiwan. Participants were high school students aged 13 to 18 years. Data were weighted and analysed using SUDAAN 11.0 and SAS 9.4. Participants were asked about intake frequencies of SSB and were grouped into four different SSB intake groups based on the combination of high or low frequency (including moderate frequency) of intake of sweetened tea and soda/sports/energy drinks. Results indicated over $99 \%$ of teens reported having at least one SSB in the past week. Smoking status was significantly associated with SSB intake types with high tea intake (high tea and low soda (HL) group, OR 7.56, $P<0.001$; high tea and high soda (HH) group, OR 9.96, $P<0 \cdot 001)$. After adjustment for potential confounders, adolescents in the low tea and high soda (LH) group $(\beta=0 \cdot 05, P=0.034)$ had significantly higher mean serum uric acid values. In conclusion, sugary tea remains the SSB of choice for Taiwanese adolescents. Those with a frequent intake of soda/sports/ energy drinks had a higher chance of being hyperuricaemic.

Key words: Sugar-sweetened beverages: Food frequency questionnaires: Serum uric acid: Adolescents: Nutrition and Health Survey in Taiwan

Overconsumption of sugar-sweetened beverages (SSB) increases the risk of non-communicable diseases including obesity, gout, CVD, fatty liver, insulin resistance and diabetes $^{(1,2)}$. Past research has found that adolescents are more likely to have greater intake of SSB than children and adults $^{(3-5)}$ and that SSB provide about $20-25 \%$ of total sugar intake $e^{(6,7)}$. Despite a decline in SSB consumption by adolescents in a number of countries (including Austria, UK and USA) ${ }^{(8-11)}$, consumption remains high. For example, SSB still provided $9 \cdot 3-9.7 \%$ of total energy intake by US adolescents from 2011 to $2014^{(12)}$ and $14 \%$ of total energy intake by UK adolescents from $2008^{(9)}$. The contribution of SSB could be close to or exceeding $10 \%$ which is recommended by the 2015 WHO guidelines ${ }^{(13)}$. The SSB intake rate is very

Abbreviations: HFCS, high-fructose corn syrup; HH, high tea ( $\geq 7$ times/week) and high soda/sports drink intake ( $\geq 4.5$ times/week); HL, high tea $(\geq 7$ times/week) and low soda/sports drink intake ( $<4.5$ times/week); LH, low tea ( $<7$ times/week) and high soda/sports drink intake $(\geq 4.5$ times/week); LL, low tea $(<7$ times/week) and low soda/ sports drink intake ( $<4.5$ times/week); NAHSIT, Nutrition and Health Survey in Taiwan; SSB, sugar-sweetened beverages.

* Corresponding author: H. Y. Chang, fax +886 37 586261, email hsingyi@nhri.org.tw 
high in Asia; for example, it reached $38 \%$ in $\mathrm{Korea}^{(14)}$ and reached $87.7 \%$ in Taiwan in $2009^{(15)}$. Besides, SSB contributed $25 \%$ of daily intakes of sugar in $\operatorname{Japan}^{(7)}$.

An important contributing factor to SSB consumption in Taiwan is its availability. Sweetened beverages are readily obtained from convenience stores as well as beverage shops including cafés, juice stores and tea shops. By 2017, there were an estimated 10662 convenience stores belonging to the four major chains and 21346 beverage shops, which equates to an average of one convenience store per 2211 persons and one beverage shop per 1104 persons $^{(16,17)}$. The greater the number of such stores near schools and the shorter walking distance required, the greater the mean daily frequency of SSB purchasing by adolescents ${ }^{(18)}$.

Although a large number of studies have examined SSB intake in adolescents, most are from Western countries and have focused on intake of carbonated drinks (including soda, carbonated soda and carbonated beverages) $)^{(3,19-21)}$. Culture plays an important role in the types of beverages consumed by adolescents, with intake of beverages (including coffee and tea) more common in adolescents in Asia and even surpassing that of soda is some Asian countries ${ }^{(19)}$. A survey conducted in 2015 in southern Taiwan showed that SSB contributed $83.5 \%$ of sugar intake, mainly sweetened tea or milk tea $(41 \%)$ in teenagers (13-18 years of age ${ }^{(22)}$. On average, sweetened tea in Taiwan contains $9.19 \mathrm{~g} / 100 \mathrm{ml}$ sugar ${ }^{(23)}$. Normally, servings come in medium $(500 \mathrm{ml})$ or large $(700 \mathrm{ml})$ sizes. If a teenager consumes $9623 \mathrm{~kJ} / \mathrm{d}$ (2300 kcal/d), about $10 \%$ of the total energy would come from a glass of sweetened tea.

Different types of SSB have different associations with health outcomes. One cross-sectional study showed that excessive intakes of sweetened coffee/tea were associated with larger BMI, waist circumference, and uric acid, whereas the association was not observed in other soft drinks ${ }^{(24)}$. Another study on children aged 6-13 years found that sweetened tea, soft drinks and sports drinks were associated with obesity. There was found to be an association of intakes of sweetened tea with central obesity (OR 1.55; $95 \%$ CI 1.26, 1.90). But this association was not observed in those who drank coffee ${ }^{(25)}$. A study on girls found that drinking soda and sports drinks was positively associated with the amount of fructose intake. On the other hand, drinking coffee/tea was associated with the amount of sucrose intake ${ }^{(26)}$. Besides, beverage shops, which sell hand-made beverages mostly, increased sharply in Taiwan. Therefore, we separated the types of SSB for analyses.

As the above research shows, SSB are an important potential health risk for Taiwanese adolescents. Moreover, it emphasises the importance of separating 'tea' as a unique beverage type in this setting. The aim of the present study was to examine changes in SSB consumption by adolescents in Taiwan over 18 years using nationally representative survey data, and to examine associated changes in anthropometric measures and blood biochemistry. Tea beverages were examined as a unique category and participants were grouped into types of SSB consumption based on high or low intakes of sweetened tea and soda/sports/energy drinks.

\section{Experimental methods}

\section{Participants}

The present study analysed secondary data. Two separate cross-sectional datasets for the present study come from the 1993-1996 and 2010-2011 Nutrition and Health Survey in Taiwan (NAHSIT), whose youth data are available for application. The sampling population for the 1993-1996 survey was Taiwanese residents aged 3 years and over with a survey completion rate of $74 \%$. In the 2010-2011 survey, the sample population was high school students from public and private schools with a completion rate of $87 \%$ in junior high school students (similar to grades 7 to 9; age range $12-15$ years old) and $92 \%$ in senior high school students (similar to grades 10 to 12; age range 15-18 years old). Further details about survey participants and sampling method have been described previously ${ }^{(27,28)}$. The present study includes adolescents at junior or senior high school aged 13 to 18 years. Adolescents aged 12 years were excluded because of incomplete data. Data collected included a FFQ, anthropometry and blood samples. Extreme values that were over the lower or upper outer fence in box plots were removed ( $n$ 186, 4.17\%), which included excessively high $(\geq 41840 \mathrm{~kJ})$ or low $(<836.8 \mathrm{~kJ})$ daily energy intake ( $n$ 10). Each drink had its extreme value, e.g. coffee $>10$ times/week or tea $>50$ times/week. SSB intake frequencies ( $n$ 64) or outlying blood results ( $n$ 109) were excluded; a total of 4277 participants were included in the analysis (1993-1996 survey, $n$ 1788; 2010-2011 survey, $n$ 2489).

The present study was conducted according to the guidelines laid down in the Declaration of Helsinki and all procedures involving human subjects were approved by the National Health Research Institutes Research Ethics Committee (ethics no. EC 0990803-R2, EC1000311-R1, EC1060110-E). Written informed consent was obtained from all subjects in 2010-2011 and verbal informed consent was obtained in 1993-1996 (ethical approval was not required in 1993-1996). Verbal consent was witnessed and formally recorded.

\section{Measurements}

Intake of different types of SSB was assessed using a FFQ ${ }^{(29)}$ which asked participants about their frequency of intake of five types of beverages in the past month (every day, every week, every month) including tea, soda/sports/energy drinks, fruit drinks (including $<100 \%$ pure juice drinks such as reconstituted and fermented juice), coffee, and other beverages (yogurt drinks, smoothies, shakes and Slurpee). For ease of analysis, we converted all intake frequencies to a proportional intake per week. The five beverage types did not include 100 $\%$ pure fruit juice or alcoholic beverages. The mean weekly intake and prevalence of consumption of each beverage type were compared for the two survey time points. We then categorised participants into four groups based on high or low levels of intake of the two most commonly consumed beverage types of tea and soda/sports/energy drinks. High ( $\geq 7$ times/week for tea and $\geq 4.5$ times/week for soda/ sports/energy drinks) and low or moderate $(<7$ times/week 
for tea and $<4.5$ times/week for soda/sports/energy drinks) intake frequencies were defined using the 3rd quartile frequency in 1993-1996. This approach enabled examination of the combined effects of these two beverage types which we believe leads to more accurate results. The resulting four SSB intake groups were: low intake of tea drinks and low intake of soda/sports/energy drinks (LL group), low intake of tea drinks and high intake of soda/sports/energy drinks (LH group), high intake of tea drinks and low intake of soda/sports/energy drinks (HL group), and high intake of both tea drinks and soda/sports/energy drinks (HH group).

Anthropometric measures and blood biochemistry were collected as part of the NAHSIT survey. Anthropometric measures include height, weight, BMI and waist circumference. BMI was calculated as weight in $\mathrm{kg}$ divided by height in $\mathrm{m}^{2}$. BMI categories was based on the criteria developed by the Health Promotion Administration, Ministry of Health and Welfare. There were different cut-off points for males and females at different ages. Participants were classified into underweight, normal-weight, overweight, and obese ${ }^{(30)}$. Waist circumference was measured at the midpoint between the top of the pelvis and the bottom of the ribs with the participant standing. Venous blood samples were taken from participants after $8 \mathrm{~h}$ of fasting. Blood samples were collected in vacutainer tubes and after being centrifuged were stored on dry ice. Then, they were delivered to a central laboratory and frozen at $-70^{\circ} \mathrm{C}$ on the same day for further analyses. Blood results included HDL-cholesterol, LDL-cholesterol, total cholesterol, TAG, fasting blood glucose and serum uric acid.

Other variables obtained from the NAHSIT questionnaire included age, sex, smoking status (never smoker, ever-smoker includes ex-smoker, occasional smoker and current smoker), alcohol consumption (never drinker, ever-drinker - mean intake of $>0$ drinks per month), physical activity and total energy intake. Physical activity was converted into weekly metabolic equivalents (MET) based on the method proposed by Ridley $e t a l .^{(31)}$. Because physical activity and total energy intake were heavily skewed to the right, they were dichotomised into low or high comparing the median of 19931996. Physical activity was classified into low $(<125.5 \mathrm{~kJ} / \mathrm{kg}$ per h) or high $(\geq 125.5 \mathrm{~kJ} / \mathrm{kg}$ per h), and total energy intake was classified into low $(<7385 \mathrm{~kJ} / \mathrm{d})$ or high $(\geq 7385 \mathrm{~kJ} / \mathrm{d})$. FFQ also asked about taking meals regularly. If a person answered to taking three meals daily, this person was considered having a regular dietary habit.

\section{Statistical analyses}

Statistical analyses and adjustment for unequal sampling rates were carried out using SAS version 9.4 (SAS Institute Inc.) and SUDAAN version 11.0. The samples were also weighted to make them representative of the population for the given year. Weekly intakes of beverages were described using mean values and $95 \%$ CI. Percentages were used to describe the prevalence of consumption of each beverage type as well as the distribution of participant characteristics by SSB intake type. Also, $t$ tests were used to analyse differences in mean intakes between surveys and $\chi^{2}$ tests were used to compare differences in prevalence between surveys and differences in the distribution of characteristics by SSB intake group. Multinomial logistic regression was used to examine participant characteristics associated with SSB intake group. Multiple linear regression was used to examine associations between SSB intake group and anthropometric measures and blood results after controlling for survey year, age, sex, metabolic equivalents (MET) and total energy intake. As only LDL-cholesterol, carbohydrate, lipid and SFA had a normal distribution, other measures were all $\log$ transformed before being entered into models. After removal of outliers and log transformation, the degree of skewness of residuals in regression models approached zero and kurtosis was less than an absolute value of 2 . Two-sided $P$ values $<0.5$ were considered statistically significant.

We compared the differences between the removed ( $n$ 183) and the rest $(n$ 4277) and found no differences in age, sex and weight. We also conducted sensitivity analysis comparing the results with ( $n$ 4460) and without the outliers ( $n$ 4277). We obtained similar coefficients, but the variation was larger in the dataset with outliers. For example, the OR were 0.054 $(95 \%$ CI $0.00,0.10) v .0 .040(95 \%$ CI $-0.03,0.11)$ for $\log$-serum uric acid without and with the outliers, respectively.

\section{Results}

Table 1 shows the distribution of participant characteristics in both surveys after weighting. Compared with those participating in survey 1, more junior high school students responded in survey $2(P=0 \cdot 001)$. In addition, survey 2 participants were less likely to be underweight and more likely to be obese $(P=0 \cdot 001)$, were more likely to have excessive total energy intakes $(P<0.001)$, and were more likely to not eat lunch regularly $(P=0.006)$.

Table 2 shows the mean frequency and prevalence of intake of each SSB group age standardised to the age distribution in 1993-1996. In the 1993-1996 survey, adolescents had a mean frequency of SSB intake of 12.4 times per week and as many as $99.1 \%$ of adolescents drank at least one SSB per week. The mean SSB intake frequency declined to 10.8 times per week in the 2010-2011 survey $(P=0.04)$; however, $99.5 \%$ of adolescents still reported consuming at least one SSB per week. Both the frequency and prevalence of intake were highest for tea drinks in both surveys, followed by soda/sports/energy drinks and other drinks in 1993-1996, and by other drinks and soda/ sports/energy drinks in 2010-2011. Taiwanese adolescents consumed tea drinks an average of four times per week in 1993-1996 and this further increased to five times per week in 2010-2011. In contrast, the intake frequency of both soda/sports/energy drinks and fruit drinks more than halved during the same period $(P<0.001)$. The prevalence of tea consumption also increased from $86.4 \%$ in 1993-1996 to $94.9 \%$ in 2010-2011 ( $P=0.005)$, whereas that of soda/sports/energy drinks decreased from 79.9 to $76.3 \%(P=0.070)$ and that of fruit drinks decreased from $60 \cdot 3$ to $51 \cdot 8 \%(P=0.015)$. Prevalence of drinking coffee and other drinks increased significantly. Prevalence increased from 33.2 to $40.3 \%(P=0 \cdot 027)$ 
Table 1. Characteristics of participants in both survey years ( $n$ 4277) (Numbers of participants and weighted percentages)

\begin{tabular}{|c|c|c|c|c|c|}
\hline \multirow[b]{2}{*}{ Characteristic } & \multicolumn{2}{|c|}{ 1993-1993 ( $n$ 1788) } & \multicolumn{2}{|c|}{ 2010-2011 ( $n$ 2489) } & \multirow[b]{2}{*}{$P^{*}$} \\
\hline & $n$ & Weighted \% & $n$ & Weighted \% & \\
\hline Age group ( $n$ 4277) & & & & & 0.001 \\
\hline Young (junior high school) & 1110 & 49.5 & 1444 & $64 \cdot 3$ & \\
\hline Older (senior high school) & 678 & 50.5 & 1045 & $35 \cdot 7$ & \\
\hline Sex $(n$ 4277) & & & & & 0.207 \\
\hline Male & 894 & 53.2 & 1229 & $51 \cdot 7$ & \\
\hline Female & 894 & $46 \cdot 8$ & 1260 & $48 \cdot 9$ & \\
\hline BMI categories $(n$ 3255) $\dagger$ & & & & & 0.001 \\
\hline Underweight & 106 & $12 \cdot 5$ & 127 & $5 \cdot 3$ & \\
\hline Normal weight & 758 & 69.0 & 1436 & 65.9 & \\
\hline Overweight & 106 & $11 \cdot 0$ & 292 & 13.4 & \\
\hline Obese & 77 & 7.5 & 353 & $15 \cdot 3$ & \\
\hline Smoking status ( $n$ 3254) & & & & & 0.221 \\
\hline Non-smoker & 1193 & $89 \cdot 3$ & 1729 & $92 \cdot 1$ & \\
\hline Ever-smokerł & 139 & $10 \cdot 7$ & 193 & 7.9 & \\
\hline Alcohol drinking status ( $n$ 3471) & & & & & 0.662 \\
\hline Non-drinker & 1213 & $80 \cdot 3$ & 1515 & 79.9 & \\
\hline Ever-drinker & 336 & $19 \cdot 7$ & 407 & $20 \cdot 1$ & \\
\hline MET/week (kJ/kg per h) (n 3433) & & & & & 0.079 \\
\hline Low $(<125 \cdot 5)$ & 726 & $50 \cdot 1$ & 1058 & $55 \cdot 3$ & \\
\hline High ( $\geq 125.5)$ & 797 & 49.9 & 852 & 44.7 & \\
\hline Total energy $(\mathrm{kJ} / \mathrm{d})(n$ 4277) & & & & & $<0.001$ \\
\hline Low $(<7385)$ & 967 & $50 \cdot 1$ & 726 & $28 \cdot 8$ & \\
\hline High $(\geq 7385)$ & 821 & $49 \cdot 9$ & 1763 & $71 \cdot 2$ & \\
\hline \multicolumn{6}{|l|}{ Dietary behaviour } \\
\hline Eats breakfast ( $n$ 4249) & & & & & 0.064 \\
\hline Irregularly (<7 d/week) & 556 & 28.4 & 862 & $33 \cdot 2$ & \\
\hline Regularly (=7 d/week) & 1205 & $71 \cdot 6$ & 1626 & $66 \cdot 8$ & \\
\hline Eats lunch ( $n$ 4250) & & & & & 0.006 \\
\hline Irregularly (<7 d/week) & 208 & $10 \cdot 8$ & 456 & $16 \cdot 7$ & \\
\hline Regularly (=7 d/week) & 1554 & $89 \cdot 2$ & 2032 & $83 \cdot 3$ & \\
\hline Eats dinner ( $n$ 4249) & & & & & 0.505 \\
\hline Irregularly (<7 d/week) & 169 & $10 \cdot 1$ & 311 & $11 \cdot 3$ & \\
\hline Regularly (=7 d/week) & 1592 & 89.9 & 2177 & $88 \cdot 7$ & \\
\hline
\end{tabular}

MET, metabolic equivalents.

${ }^{*} P$ value based on the $\chi^{2}$ test.

† BMI was calculated as weight in kg divided by height in metres squared. BMl categories were based on the criteria developed by the Health Promotion Administration, Ministry of Health and Welfare. There were different cut-off points for males and females at different ages. Participants were classified into underweight, normal-weight, overweight, and obese $^{(30)}$.

‡ Ever-smoker: including former smokers and current smokers. Ever-drinker: consumes alcohol >0 times per month.

and from 76.4 to $85.3 \%(P=0.032)$ for coffee and other drinks, respectively.

Participants were grouped into four SSB intake groups based on whether they had low and moderate ( $\mathrm{L}$ group) or high ( $\mathrm{H}$ group) intake of tea and soda/sports/energy drinks (data not shown). The cut-off point was the 3rd quartile of that drink. It was seven times/week for tea, and 4.5 times/ week for soda/sports/energy drinks. The most common intake group in adolescents in both surveys was low in tea and low in soda drink intake (LL) (from 57.8 to $64.8 \%$ of adolescents), followed by high in tea and low in soda drink intake (HL) (from 17.3 to $25.2 \%$ ). The prevalence in low in tea and high in soda drink (LH) and high in both groups $(\mathrm{HH})$ decreased significantly over 18 years. Prevalence dropped from 14.4 and $10.4 \%$ in 1993-96 to 5.1 and 4.9 $\%$ in 2010-11 $(P<0 \cdot 001)$ for $\mathrm{LH}$ and $\mathrm{HH}$, respectively.

Table 3 shows the results of multinomial logistic regression analyses examining associations between participant characteristics and the four SSB intake groups. There were significantly fewer adolescents with LH intake (OR 0.20; $P<0.001)$ and
HH intake (OR 0.33; $P=0.019)$ compared with the LL group in 2010-2011. Being an ever-smoker (includes current smokers) was associated with a greater likelihood of HL intake (OR 7.56; $P<0.001)$ and $\mathrm{HH}$ intake (OR 9.96; $P<0.001)$. Having high total energy intake (OR 2.50; $P=0.017$ ) was also associated with a greater likelihood of $\mathrm{HH}$ intake. Not eating breakfast regularly was associated with a greater likelihood of LH intake (OR 1.73; $P=0.002$ ).

Table 4 shows the mean values of anthropometric measures and blood results and different mean values between the two surveys by SBB intake group. All values in Table 4 were adjusted by age prevalence in 1993-1996. Total cholesterol was significantly lower in the $\mathrm{HH}$ group compared with the HL group in 1993-1996 ( $<<0.05)$, and fasting blood glucose was significantly higher in the LH group compared with the LL group in 2010-2011 $(P<0 \cdot 05)$. Serum uric acid was highest in the LH group, followed by the HH group in both surveys, and the lowest value was in the LL group. All the comparisons showed that uric acid in $\mathrm{LH}$ or $\mathrm{HH}$ was higher than that in LL $(P<0.05)$, and it was higher in LH than in 
Table 2. Weekly frequency of intake and prevalence of consumption of each type of sugar-sweetened beverage (SSB) in both survey years $(n 4277)^{*}$ (Mean values and $95 \%$ confidence intervals; percentages of consumers)

\begin{tabular}{|c|c|c|c|c|c|c|c|c|}
\hline \multirow[b]{3}{*}{ Category } & \multicolumn{3}{|c|}{ 1993-1996 (n 1788) } & \multicolumn{3}{|c|}{ 2010-2011 (n 2489) } & \multirow[b]{3}{*}{$P$ for mean $\dagger$} & \multirow[b]{3}{*}{$P$ for $\% \neq$} \\
\hline & \multicolumn{2}{|c|}{ Times/week } & \multirow[b]{2}{*}{ Consumer (\%) } & \multicolumn{2}{|c|}{ Times/week } & \multirow[b]{2}{*}{ Consumer (\%) } & & \\
\hline & Mean & $95 \% \mathrm{Cl}$ & & Mean & $95 \% \mathrm{Cl}$ & & & \\
\hline SSB & $12 \cdot 4$ & $11,13.8$ & 99.1 & $10 \cdot 8$ & $10 \cdot 2,11.4$ & 99.5 & 0.040 & 0.230 \\
\hline Tea & $4 \cdot 3$ & $3 \cdot 6,5 \cdot 0$ & $86 \cdot 4$ & $5 \cdot 1$ & $4 \cdot 7,5 \cdot 5$ & 94.9 & 0.041 & 0.005 \\
\hline Soda/sports/energy drinks & 3.4 & $3 \cdot 1,3 \cdot 7$ & 79.9 & $1 \cdot 7$ & $1.6,1.9$ & $76 \cdot 3$ & $<0.001$ & 0.070 \\
\hline Fruit drinks & 1.7 & $1 \cdot 4,2 \cdot 0$ & $60 \cdot 3$ & 0.8 & $0.7,0.9$ & $51 \cdot 8$ & $<0.001$ & 0.015 \\
\hline Coffee & 0.5 & $0.4,0.7$ & 33.2 & 0.6 & $0.5,0.7$ & $40 \cdot 3$ & 0.441 & 0.027 \\
\hline Other drinks & 2.5 & $2 \cdot 0,3 \cdot 0$ & $76 \cdot 4$ & 2.5 & $2 \cdot 2,2 \cdot 9$ & 83.5 & 0.886 & 0.032 \\
\hline
\end{tabular}

*Values were age standardised to the age distribution of 1993-1996. Soda/sports/energy drinks included soda, sports drinks and energy drinks; fruit drinks refer to non-100 \% juice drinks including reconstituted juice and fermented juice; other drinks included yogurt drinks, smoothies, shakes and Slurpee.

† $P$ value based on the $t$ test for the comparison of means between 2010-2011 and 1993-1996.

$\ddagger P$ value based on the $\chi^{2}$ test for the comparison of proportions between 2010-2011 and 1993-1996.

HL $(P<0 \cdot 05)$. In addition, uric acid level in HH was higher than that in HL in 2010-2011 $(P<0 \cdot 05)$. Statistically significant increases in BMI, waist circumference and fasting blood glucose $(P<0.001)$ were observed between the two surveys. Serum uric acid significantly decreased $(P<0.001)$ compared with the first survey. BMI, waist circumference and fasting blood glucose were significantly different in the four different drinking groups $(P<0 \cdot 05)$.

Table 5 shows the associations between the four SSB intake groups and anthropometric measures and blood biochemistry results. After adjustment for potential confounders, compared with the LL group, adolescents in the LH group $(\beta=0 \cdot 05$; $P=0.032)$ had significantly higher mean serum uric acid values. No other significant associations were observed between SSB intake group and anthropometric measures or blood results. We also examined serum uric acid values stratifying by sex (data not shown) and found that serum uric acid levels were significantly lower in both males and females $(P<0.001)$ in the second survey. Mean serum uric acid decreased from 7.4 to $6.7 \mathrm{mg} / \mathrm{dl}(440.2$ to 398.5 $\mu \mathrm{mol} / \mathrm{l}$ ) in males, and from 5.5 to $4.8 \mathrm{mg} / \mathrm{dl}$ (327.1 to $285.5 \mu \mathrm{mol} / 1$ in females. In both surveys, mean serum uric acid was significantly higher in males compared with females in all of the four SSB intake groups $(P<0 \cdot 05)$. In the adjusted analysis, no significant differences in serum uric acid between SSB intake groups were observed in males and females.

\section{Discussion}

We observed a decline in frequency of intake of SSB in adolescents over a 14-year period. However, the prevalence of SSB consumption remains high with over $99 \%$ of adolescents consuming at least one type of SSB on a weekly basis. In addition, the prevalence of sweetened tea intake significantly increased and the mean frequency of intake of sweetened tea drinks was as high as five times per week. These findings confirm the importance of measuring tea as a separate beverage type in our population. Frequent consumption of sweetened tea drinks with occasional consumption of soda/sports/ energy drinks was the most common intake type ( $43 \%$ of adolescents in the 2010-2011 survey) and also demonstrated the largest increase in prevalence over time. Smoking status was the chance factor most strongly associated with high SSB intake. In adjusted analyses of anthropometric measures and blood biochemistry, serum uric acid levels were higher in adolescents who frequently consumed soda/sports drinks (regardless of high or low tea drink consumption).

Despite the decline in mean frequency, the prevalence of SSB consumption in our study was high. The prevalence of daily SSB consumption in children and adolescents decreased from $79.7 \%$ in $2003-2004$ to $60.7 \%$ in 2014 in the USA ${ }^{(32)}$, and from $76.8 \%$ in 2003 to $71.4 \%$ in 2008 in Australia ${ }^{(8)}$. These trends in SSB intake are probably related to declines in soda drink consumption, energy intake from soda drinks, and the proportion of total energy intake from soda drinks since the year 2000. In contrast, consumption of other beverages such as coffee and tea or sports and energy drinks has increased ${ }^{(8,10,11)}$. In our study, we found that the prevalence of weekly tea intake significantly increased from 94.5 to $99.1 \%$ whereas that of soda/sports/energy drinks decreased from 79.9 to $76.3 \%$. Similar results have been found in Korea, where the prevalence of drinking soda drinks has declined from $22 \%$ in 2001 to $18 \%$ in 2009 in adolescents. However, the prevalence of overall SSB intake has increased due to increased intake of other beverages (including sports drinks/energy drinks, coffee/tea, flavoured milk and other drinks $)^{(14)}$. A similar phenomenon has been observed in Mexico, where despite the prevalence of SSB consumption increasing from $91.5 \%$ in 1999 to $94 \%$ in 2012, there has been no statistically significant change in the prevalence of soda drink consumption. In contrast, the prevalence of sugar-sweetened coffee or tea intake has significantly increased from $14 \cdot 2$ to $20 \cdot 8 \%{ }^{(33)}$.

Smoking consumption in adolescents can be associated with intake of $\operatorname{SSB}^{(34,35)}$. In our study, smoking status was the only participant characteristic significantly associated with two different SSB intake groups. Both of the intake groups associated with smoking ( $\mathrm{HL}$ and $\mathrm{HH}$ ) involved frequent consumption of tea drinks, indicating that those who frequently drink tea beverages are also more likely to smoke. If frequent consumption of soda/sports drinks is added to frequent tea drink consumption, then the likelihood of smoking further increases 


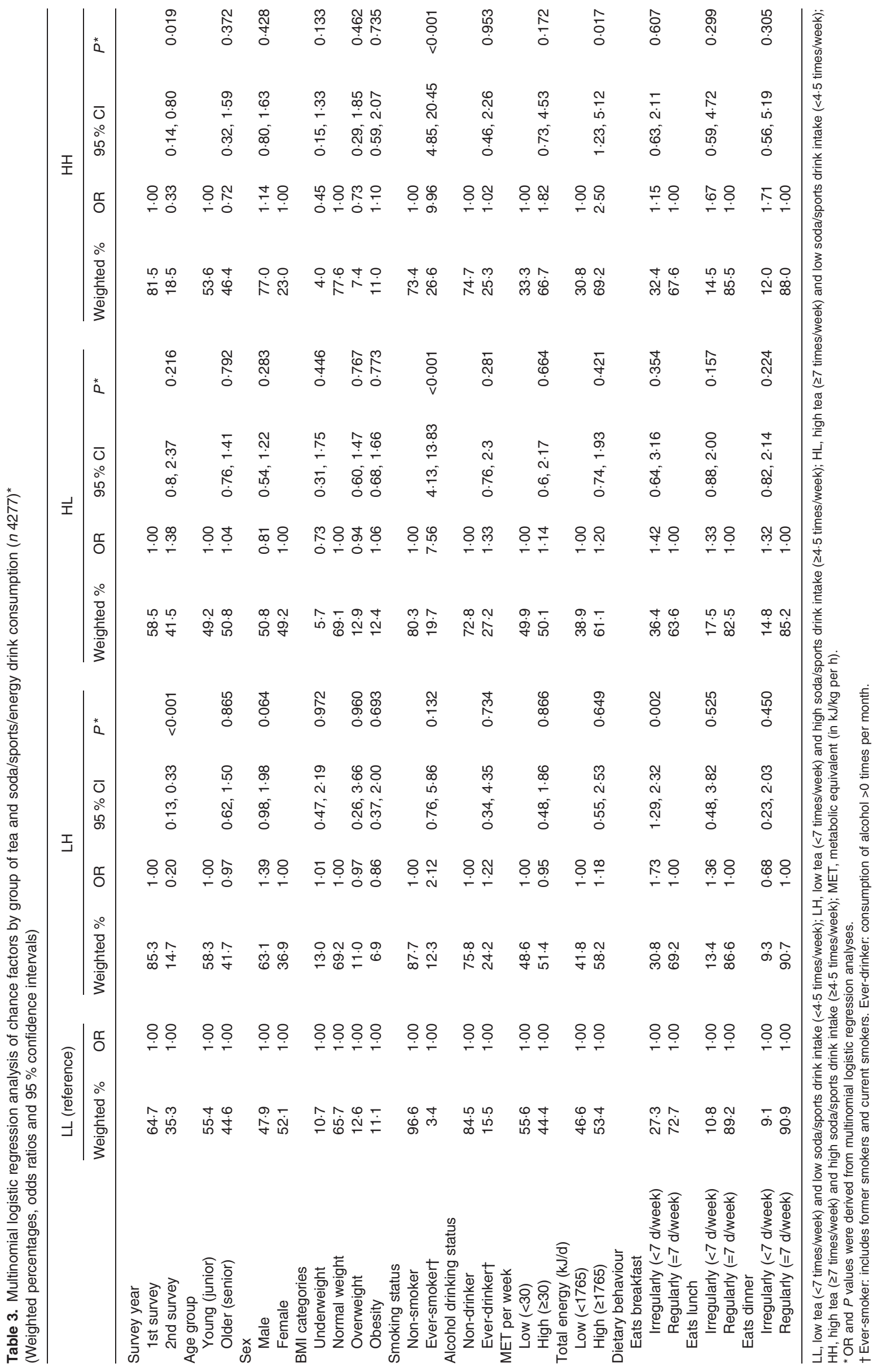


Table 4. Distribution of anthropometric measures and blood biochemistry by group of tea and soda/sports/energy drink consumption in adolescents $(n$ 4277)*

(Mean values or mean differences with their standard errors)

\begin{tabular}{|c|c|c|c|c|c|c|c|c|c|c|c|c|c|c|c|}
\hline \multirow[b]{2}{*}{ Variable } & \multicolumn{3}{|c|}{ All } & \multicolumn{3}{|c|}{ LL } & \multicolumn{3}{|c|}{$\mathrm{LH}$} & \multicolumn{3}{|c|}{$\mathrm{HL}$} & \multicolumn{3}{|c|}{$\mathrm{HH}$} \\
\hline & Mean & SE & $P$ & Mean & SE & $P$ & Mean & SE & $P$ & Mean & SE & $P$ & Mean & SE & $P$ \\
\hline \multicolumn{16}{|c|}{ 1993-1996 (1st survey) } \\
\hline BMI $\left(\mathrm{kg} / \mathrm{m}^{2}\right)$ & 20.2 & 0.1 & & $20 \cdot 2$ & 0.3 & & $19 \cdot 6$ & 0.3 & & $20 \cdot 4$ & 0.3 & & $20 \cdot 3$ & 0.4 & \\
\hline WC $(\mathrm{cm})$ & 65.6 & 0.3 & & $65 \cdot 8$ & 0.6 & & 64.9 & $1 \cdot 1$ & & $65 \cdot 3$ & 0.7 & & $66 \cdot 3$ & 1.5 & \\
\hline $\mathrm{HDL}-\mathrm{C}(\mathrm{mg} / \mathrm{dl}) \|$ & 57.6 & 1.6 & & 57.6 & 1.1 & & 55.4 & 4.6 & & $60 \cdot 1$ & 2.3 & & $56 \cdot 6$ & 2.2 & \\
\hline LDL-C (mg/dl)\| & $90 \cdot 1$ & 1.7 & & 89.1 & 2.5 & & 94.9 & $2 \cdot 8$ & & 91.5 & 3.1 & & 85.2 & 4.6 & \\
\hline TC (mg/dl)\| & 161.5 & $2 \cdot 1$ & & $160 \cdot 6$ & 2.3 & & 164.1 & 3.1 & & $165 \cdot 3$ & 3.6 & & 154.7 & $5 \cdot 3$ & $\S$ \\
\hline TAG (mg/dl)\| & 68.3 & $2 \cdot 0$ & & 68.8 & 2.6 & & 68.8 & $3 \cdot 3$ & & 67.9 & 2.0 & & 63.4 & 4.2 & \\
\hline FBG $(\mathrm{mg} / \mathrm{dl}) \|$ & $80 \cdot 0$ & $0 \cdot 8$ & & $80 \cdot 0$ & 0.8 & & 79.9 & 0.7 & & 79.7 & 1.3 & & 80.5 & 0.7 & \\
\hline SUA (mg/dl)\| & 6.5 & 0.1 & & $6 \cdot 3$ & 0.1 & & $7 \cdot 0$ & 0.2 & $\dagger$ & 6.4 & 0.1 & $\ddagger$ & $6 \cdot 8$ & 0.2 & $\dagger$ \\
\hline \multicolumn{16}{|c|}{ 2010-2011 (2nd survey) } \\
\hline $\mathrm{BMl}\left(\mathrm{kg} / \mathrm{m}^{2}\right)$ & 21.6 & 0.1 & & 21.5 & 0.1 & & $22 \cdot 2$ & 0.5 & & $21 \cdot 7$ & 0.2 & & $21 \cdot 8$ & 0.5 & \\
\hline WC (cm) & $76 \cdot 7$ & 0.3 & & $76 \cdot 2$ & 0.4 & & $78 \cdot 1$ & 1.3 & & 77.4 & 0.5 & & 77.4 & $1 \cdot 1$ & \\
\hline $\mathrm{HDL}-\mathrm{C}(\mathrm{mg} / \mathrm{dl})$ & $55 \cdot 2$ & 0.4 & & $55 \cdot 7$ & 0.5 & & $52 \cdot 8$ & 1.5 & & 54.7 & 0.5 & & 52.5 & 1.8 & \\
\hline LDL-C (mg/dl) & $90 \cdot 3$ & 0.8 & & $89 \cdot 8$ & 1.0 & & $96 \cdot 1$ & $3 \cdot 1$ & & $90 \cdot 1$ & 1.4 & & 93.2 & 2.5 & \\
\hline $\mathrm{TC}(\mathrm{mg} / \mathrm{dl})$ & 159.7 & 0.9 & & 159.6 & $1 \cdot 2$ & & 164.4 & $2 \cdot 7$ & & 159.1 & 1.4 & & $160 \cdot 8$ & $2 \cdot 2$ & \\
\hline TAG (mg/dl) & 71.3 & $1 \cdot 1$ & & $70 \cdot 3$ & $1 \cdot 1$ & & $78 \cdot 3$ & 3.9 & & 72.4 & 1.7 & & 74.1 & 3.6 & \\
\hline FBG (mg/dl) & 94.6 & 0.4 & & 94.3 & 0.4 & & 97.4 & 1.4 & $\dagger$ & 94.8 & 0.6 & & 94.5 & $1 \cdot 1$ & \\
\hline SUA (mg/dl) & $5 \cdot 8$ & 0.0 & & $5 \cdot 7$ & 0.0 & & 6.6 & 0.2 & $\dagger$ & $5 \cdot 9$ & 0.1 & † & $6 \cdot 3$ & 0.1 & $\dagger \S$ \\
\hline \multicolumn{16}{|c|}{ Mean difference (2nd survey-1st survey) } \\
\hline BMI $\left(\mathrm{kg} / \mathrm{m}^{2}\right)$ & 1.4 & 0.2 & $<0.001$ & $1 \cdot 2$ & 0.3 & 0.003 & $2 \cdot 6$ & 0.5 & 0.001 & $1 \cdot 2$ & 0.4 & 0.008 & 1.5 & 0.6 & 0.041 \\
\hline WC $(\mathrm{cm})$ & 11 & 0.5 & $<0.001$ & $10 \cdot 4$ & 0.7 & $<0.001$ & $13 \cdot 2$ & 1.7 & $<0.001$ & $12 \cdot 1$ & 0.8 & $<0.001$ & 11.1 & 1.9 & $<0.001$ \\
\hline HDL-C (mg/dl) & -2.5 & 1.7 & 0.164 & -1.9 & $1 \cdot 2$ & 0.151 & $-2 \cdot 6$ & 4.8 & 0.605 & $-5 \cdot 4$ & $2 \cdot 4$ & 0.045 & -4.0 & 2.9 & 0.192 \\
\hline LDL-C (mg/dl) & 0.3 & 1.9 & 0.892 & 0.7 & $2 \cdot 7$ & 0.788 & 1.3 & $4 \cdot 2$ & 0.769 & $-1 \cdot 4$ & 3.4 & 0.685 & 8.0 & $5 \cdot 2$ & 0.158 \\
\hline $\mathrm{TC}(\mathrm{mg} / \mathrm{dl})$ & $-1 \cdot 0$ & $2 \cdot 3$ & 0.452 & $-1 \cdot 0$ & 2.5 & 0.711 & 0.3 & $4 \cdot 1$ & 0.938 & $-6 \cdot 2$ & 3.8 & 0.140 & $6 \cdot 1$ & 5.7 & 0.311 \\
\hline TAG (mg/dl) & 3.1 & $2 \cdot 3$ & 0.216 & 1.4 & $2 \cdot 8$ & 0.624 & 9.5 & $5 \cdot 1$ & 0.095 & 4.6 & $2 \cdot 6$ & 0.114 & $10 \cdot 7$ & 5.5 & 0.082 \\
\hline FBG (mg/d) & $14 \cdot 6$ & 0.9 & $<0.001$ & $14 \cdot 3$ & 0.9 & $<0.001$ & 17.4 & 1.5 & $<0.001$ & $15 \cdot 1$ & 1.4 & $<0.001$ & $14 \cdot 0$ & 1.3 & $<0.001$ \\
\hline SUA (mg/dl) & -0.6 & 0.1 & $<0.001$ & -0.6 & 0.1 & $<0.001$ & -0.4 & 0.3 & 0.246 & -0.5 & 0.2 & 0.012 & -0.5 & 0.2 & 0.086 \\
\hline
\end{tabular}

LL, low tea ( $<7$ times/week) and low soda/sports drink intake ( $<4.5$ times/week); LH, low tea ( $<7$ times/week) and high soda/sports drink intake ( $\geq 4.5$ times/week); HL, high tea ( $\geq 7$ times/week) and low soda/sports drink intake ( $<4.5$ times/week); HH, high tea ( $\geq 7$ times/week) and high soda/sports drink intake ( $\geq 4.5$ times/week); WC, waist circumference; HDL-C, HDL-cholesterol; LDL-C, LDL-cholesterol; TC, total cholesterol; FBG, fasting blood glucose; SUA, serum uric acid.

* Values have been age standardised to the age distribution of 1993-1996.

† Significantly different from participants in the LL group in the same survey $(P<0.05 ; t$ test).

$\mp$ Significantly different from participants in the LH group in the same survey $(P<0.05 ; t$ test).

§ignificantly different from participants in the $\mathrm{HL}$ group in the same survey $(P<0.05 ; t$ test).

$\|$ To convert cholesterol from $\mathrm{mg} / \mathrm{dl}$ to $\mathrm{mmol} / \mathrm{l}$, multiply by 0.0259 . To convert TAG from $\mathrm{mg} / \mathrm{dl}$ to $\mathrm{mmol} / \mathrm{l}$, multiply by 0.0113 . To convert FBG from mg/dl to mmol/l, multiply by 0.0555 . To convert SUA from $\mathrm{mg} / \mathrm{dl}$ to $\mu \mathrm{mol} / \mathrm{l}$, multiply by 59.48 .

Table 5. Multivariate linear regression analysis of associations of groups of tea and soda/sports/energy drink consumption in adolescents with anthropometric measures and blood biochemistry*t

( $\beta$-Coefficients and $95 \%$ confidence intervals)

\begin{tabular}{|c|c|c|c|c|c|c|c|c|c|c|}
\hline \multirow[b]{2}{*}{ Variable } & \multirow{2}{*}{$\begin{array}{c}\mathrm{LL} \text { (reference) } \\
\beta\end{array}$} & \multicolumn{3}{|c|}{$\mathrm{LH}$} & \multicolumn{3}{|c|}{$\mathrm{HL}$} & \multicolumn{3}{|c|}{$\mathrm{HH}$} \\
\hline & & $\beta$ & $95 \% \mathrm{Cl}$ & $P$ & $\beta$ & $95 \% \mathrm{Cl}$ & $P$ & $\beta$ & $95 \% \mathrm{Cl}$ & $P$ \\
\hline Log-BMI $\left(\mathrm{kg} / \mathrm{m}^{2}\right)$ & 1.00 & -0.004 & $-0.02,0.01$ & 0.569 & -0.001 & $-0.01,0.01$ & 0.863 & 0.001 & $-0.02,0.03$ & 0.912 \\
\hline Log-WC $(\mathrm{cm})$ & 1.00 & -0.011 & $-0.05,0.03$ & 0.597 & 0.001 & $-0.01,0.01$ & 0.806 & 0.008 & $-0.02,0.04$ & 0.552 \\
\hline Log-HDL (mg/dl) & 1.00 & -0.047 & $-0.17,0.08$ & 0.420 & 0.022 & $-0.02,0.07$ & 0.309 & -0.012 & $-0.10,0.07$ & 0.753 \\
\hline $\mathrm{LDL}(\mathrm{mg} / \mathrm{dl})$ & 1.00 & 7.337 & $-1 \cdot 22,15 \cdot 89$ & 0.085 & 1.951 & $-3 \cdot 26,7 \cdot 16$ & 0.423 & 1.780 & $-3.88,7.44$ & 0.500 \\
\hline Log-TC (mg/dl) & 1.00 & 0.030 & $-0.01,0.07$ & 0.098 & 0.017 & $-0.01,0.04$ & 0.194 & 0.015 & $-0.03,0.06$ & 0.479 \\
\hline Log-TAG (mg/dl) & 1.00 & 0.035 & $-0.03,0.10$ & 0.257 & 0.011 & $-0.04,0.06$ & 0.602 & 0.024 & $-0.11,0.15$ & 0.683 \\
\hline Log-FBG (mg/dl) & 1.00 & 0.005 & $-0.01,0.01$ & 0.318 & 0.002 & $-0.01,0.02$ & 0.766 & -0.003 & $-0.03,0.02$ & 0.796 \\
\hline Log-SUA (mg/dl) & 1.00 & 0.054 & $0.00,0.10$ & 0.034 & 0.022 & $-0.01,0.06$ & 0.181 & 0.048 & $-0.02,0.11$ & 0.133 \\
\hline
\end{tabular}

LL, low tea ( $<7$ times/week) and low soda/sports drink intake ( $<4.5$ times/week); LH, low tea ( $<7$ times/week) and high soda/sports drink intake $(\geq 4.5$ times/week); HL, high tea ( $\geq 7$ times/week) and low soda/sports drink intake ( $<4.5$ times/week); $\mathrm{HH}$, high tea ( $\geq 7$ times/week) and high soda/sports drink intake ( $\geq 4.5$ times/week); WC, waist circumference; TC, total cholesterol; FBG, fasting blood glucose; SUA, serum uric acid.

* $\beta$-Coefficients are linear regression coefficients derived from multivariable linear regression analyses. All models were adjusted for survey year, age, sex, metabolic equivalent per week (in $\mathrm{kJ} / \mathrm{kg}$ per h) and total energy intake (in kJ).

$\dagger$ This Table presents results without adjusting for total energy intake. The adjusted analyses were carried out and resulted in similar coefficients.

$\ddagger$ Apart from LDL, other variables were all log transformed due to lack of a normal distribution.

substantially. Not eating breakfast regularly has been found to be associated with SSB intake ${ }^{(36-38)}$. This association was only found in the low frequent intake of tea and high frequent intake of soda/sports/energy drinks (LH) group in the present study. The association was not significant in other tea drinking groups, HL and HH. It is possible that breakfast sold in the 
stores always came with tea. Nowadays, many adolescents buy breakfast from the store instead of eating at home ${ }^{(39-41)}$.

In adjusted analyses of anthropometric measures and blood biochemistry, we found that adolescents with low frequent intake of tea and high frequent intake of soda/sports/energy drinks (LH group) had significantly higher serum uric acid levels $(P<0 \cdot 05)$. Other groups showed positive association with uric acid, but the difference did not reach statistical significance. This difference could be due to the types of sugar added to these beverages. In Taiwan, tea drinks are normally sweetened by adding sucrose (a disaccharide consisting of $50 \%$ fructose and $50 \%$ glucose) or high-fructose corn syrup (HFCS: a monosaccharide consisting of $55 \%$ fructose and $45 \%$ glucose). However, soda, sports drinks and energy drinks are sweetened mainly by adding HFCS. Although both of these sugars are formed from fructose and glucose, HFCS contains more fructose than glucose. Fructose does not have a negative feedback system via ATP and citric acid, which are involved in glucose metabolism. When fructose is stimulated by fructokinase (also known as ketohexokinase), ATP is converted to uric acid, causing a rise in its concentration $^{(42)}$. A previous study also showed that a higher intake of soda was associated with a higher level of uric acid in adults (aged above 19 years) ${ }^{(43)}$. Some studies showed that tea could reduce uric acid levels ${ }^{(44)}$. This was consistent with our finding that adolescents who drank soda more often compared with those who drank tea had high uric acid levels. On the other hand, some studies could not establish a relationship $^{(44,45)}$. However, these studies did not indicate whether sugar was added in tea. Thus, various results have been observed.

Serum uric acid levels in adolescents declined between surveys. However, mean serum uric acid in the group with the most infrequent SSB intake was $5.7 \mathrm{mg} / \mathrm{dl}(339.0 \mu \mathrm{mol} / \mathrm{l})$, which is still higher than that observed in other Asian countries such as China $(5.27 \mathrm{mg} / \mathrm{dl} ; 313.46 \mu \mathrm{mol} / \mathrm{l})^{(46)}$ and other Western countries such as the USA, Australia and Italy (4.59-5.52 mg/dl; 273.01-328.33 $\mu \mathrm{mol} / \mathrm{l}^{(47-49)}$. Serum uric acid is an independent chance factor for all-cause mortality ${ }^{(50,51)}$ and is a chance factor for childhood and adolescent chronic kidney disease ${ }^{(52)}$. Taiwan has the highest prevalence and incidence of end-stage renal disease in the world ${ }^{(53)}$. Moreover, from 2005 to 2012 the incidence of end-stage renal disease showed the largest increase in those aged 0 to 19 years $^{(54)}$. Although few studies have linked SSB to endstage renal disease ${ }^{(55,56)}$, some studies did find an association between SSB and the chance of chronic kidney disease ${ }^{(57,58)}$. As a result, control of uric acid levels and consumption of SSB are of primary importance to adolescent health in Taiwan.

The present study found that uric acid level was associated with SSB intake frequencies only after controlling for other variables. However, other studies have found that SSB intakes were associated with waist circumference ${ }^{(15,59)}, \mathrm{BMI}^{(15,59)}$, blood pressures ${ }^{(48,60)}$ and $\mathrm{TAG}^{(61)}$. This was possible due to culture difference, types of SSB, and ways of estimating SSB amount. This study only analysed the frequency of SSB intakes, and grouped SSB into two major types. That might cause the different results. Studies have shown that drinking tea could lower all-cause mortality, risks of stroke and diabetes, and improve blood pressure, total cholesterol and LDL ${ }^{(62,63)}$. Those studies were on tea without sugar. However, our study was mostly on tea drinks sold in beverage shops, which add sugar. That could cause the different results.

The strengths of the present study include the use of a large-scale dataset that was nationally representative. SSB intake was estimated using a FFQ, which facilitated understanding of the dietary habits of each participant. In addition, we compared measures taken more than 18 years apart which enabled us to better capture behavioural changes over a long time period. The present study is also the first to combine the intake frequency of two different kinds of beverages to investigate SSB intake groups. Our study also has several limitations. (1) As this is a cross-sectional analysis, we are not able to establish causal relationships. (2) As we categorised soda, sports drinks and energy drinks as one beverage type, it was not possible to examine the characteristics of those consuming only one of these types of drinks or to examine comparative changes in the consumption of these different beverages between surveys. However, we were limited by the grouping together of these three beverage types in the first NAHSIT and therefore needed to maintain this grouping when comparing results between surveys. (3) As we used FFQ, we could not estimate intake amounts. The amount of intakes can be obtained through 24-h recall. Most of the time, $1 \mathrm{~d}$ measurements were taken. The purpose of this study was to examine SSB intake types. Thus, 24-h recall data were not used. (4) In 1993-1996, the FFQ on SSB did not ask whether it was sweetened or not. At that time, shops did not label the amount of sugar added in the drink. We assumed that most adolescents selected sugar-sweetened drinks.

In conclusion, sugar-sweetened tea was the principal SSB consumed by Taiwanese adolescents. Not only was there a statistically significant increase in the prevalence of consumption of this beverage, but the mean frequency of intake increased from 4.3 times in 1993-1996 to 5 times per week in 2010-2011. High SSB intake was associated with serum uric acid and despite the decline in serum uric acid observed, the level remains high compared with other countries. Therefore, controlling SSB intake in Taiwanese adolescents remains an important public health challenge.

\section{Acknowledgements}

Part of the present study was supported by grant no. MOST-106-2320-B-400-018 from the Ministry of Science and Technology and PH-103-SP-12 from the Ministry of Health and Welfare, Taiwan. Data analysed came from the 'Nutrition and Health Survey in Taiwan (NAHSIT)' funded by the Department of Health (DOH FN8202, DOH-83-FS-41, DOH-84-FS-11, DOH-85-FS-11, DOH-86-FS-11) in 19931996 and by the Food and Drug Administration, Department of Health, Executive Yuan (99TFDA-FS-408, 100TFDAFS-406) in 2010-2011. In 1993-1996 the project was carried out by the Institute of Biomedical Sciences, Academia Sinica and the Department of Biochemistry, College of Medicine, National Taiwan University under the direction of Dr 
Wen-Harn Pan and Dr Po-Chao Huang. The Office of Nutrition Survey, Center for Survey Research, Academia Sinica was responsible for data distribution. In 2010-2011 the project was carried out by the Division of Preventive Medicine and Health Services Research, Institute of Population Health Sciences, National Health Research Institutes (NHRI) under the direction of Dr Wen-Harn Pan. The Office of Nutrition Survey, Division of Preventive Medicine and Health Services Research, Institute of Population Health Sciences, National Health Research Institutes (NHRI) are responsible for data distribution. The assistance provided by the institutes and aforementioned individuals is greatly appreciated. The views expressed herein are solely those of the authors.

Part of the present study was supported by the National Health Research Institutes (grant no. PH-103-SP-12) and the Ministry of Science and Technology (grant no.105-3011-F-400-001). The content of this paper reflects only the authors' views. The sponsors did not place any restrictions on the study design.

The authors' responsibilities were as follow: Y. H. S. analysed the data and drafted the manuscript; H. Y. C. conceptualised the study, supervised the analysis and revised the manuscript; H. C. W. was responsible for the intake data and provided a critical review of the manuscript; F. F. S. provided critical review of the manuscript; W. H. P. was responsible for the nutritional surveys and provided critical review of the manuscript; all authors read and approved the final manuscript.

The authors declare that there are no conflicts of interest.

\section{References}

1. Malik VS \& Hu FB (2015) Fructose and cardiometabolic health: what the evidence from sugar-sweetened beverages tells us. $J A m$ Coll Cardiol 66, 1615-1624.

2. Johnson RJ, Perez-Pozo SE, Sautin YY, et al. (2009) Hypothesis: could excessive fructose intake and uric acid cause type 2 diabetes? Endocr Rev 30, 96-116.

3. Ozen AE, Bibiloni Mdel M, Pons A, et al. (2015) Fluid intake from beverages across age groups: a systematic review. J Hum Nutr Diet 28, 417-442.

4. Sui Z, Zheng M, Zhang M, et al. (2016) Water and beverage consumption: analysis of the Australian 2011-2012 National Nutrition and Physical Activity Survey. Nutrients 8, E678.

5. Miller PE, McKinnon RA, Krebs-Smith SM, et al. (2013) Sugar-sweetened beverage consumption in the U.S.: novel assessment methodology. Am J Prev Med 45, 416-421.

6. Louie JC, Moshtaghian H, Rangan AM, et al. (2016) Intake and sources of added sugars among Australian children and adolescents. Eur J Nutr 55, 2347-2355.

7. Takeichi H, Taniguchi H, Fukinbara M, et al. (2012) Sugar intakes from snacks and beverages in Japanese children. J Nutr Sci Vitaminol (Tokyo) 58, 113-117.

8. Jensen BW, Nichols M, Allender S, et al. (2012) Consumption patterns of sweet drinks in a population of Australian children and adolescents (2003-2008). BMC Public Health 12, 771.

9. Ng SW, Ni Mhurchu C, Jebb SA, et al. (2012) Patterns and trends of beverage consumption among children and adults in Great Britain, 1986-2009. Br J Nutr 108, 536-551.

10. Kit BK, Fakhouri TH, Park S, et al. (2013) Trends in sugar-sweetened beverage consumption among youth and adults in the United States: 1999-2010. Am J Clin Nutr 98, 180-188.

11. Mesirow MS \& Welsh JA (2015) Changing beverage consumption patterns have resulted in fewer liquid calories in the diets of US children: National Health and Nutrition Examination Survey 2001-2010. J Acad Nutr Diet 115, 559-566.e4.

12. Rosinger A, Herrick K, Gahche J, et al. (2017) Sugar-sweetened beverage consumption among U.S. youth, 2011-2014. NCHS Data Brief 271, 1-8.

13. World Health Organization (2015) Guideline: Sugars Intake for Adults and Children. Geneva: World Health Organization.

14. Han E, Kim TH \& Powell LM (2013) Beverage consumption and individual-level associations in South Korea. BMC Public Health 13, 195.

15. Lin WT, Huang HL, Huang MC, et al. (2013) Effects on uric acid, body mass index and blood pressure in adolescents of consuming beverages sweetened with high-fructose corn syrup. Int J Obes (Lond) 37, 532-539.

16. Ministry of Economic Affairs (2018) Industrial Production Indexes in April 2018. https://www.moea.gov.tw/Mns/dos/bulletin/Bulletin. aspx?kind $=9 \& h t m l=1 \& m e n u \_i d=18808 \& b u l l \_i d=5108$ (accessed April 2018).

17. Ministry of Finance (2018) Monthly statistics of finance: January 2018. http://web02.mof.gov.tw/njswww/WebProxy.aspx?sys=100\& funid $=$ defjspf2 (accessed October 2018).

18. Hearst MO, Pasch KE \& Laska MN (2012) Urban v. suburban perceptions of the neighbourhood food environment as correlates of adolescent food purchasing. Public Health Nutr 15, 299-306.

19. Guelinckx I, Iglesia I, Bottin JH, et al. (2015) Intake of water and beverages of children and adolescents in 13 countries. Eur J Nutr 54, Suppl. 2, 69-79.

20. Abdel Rahman A, Jomaa L, Kahale LA, et al. (2018) Effectiveness of behavioral interventions to reduce the intake of sugar-sweetened beverages in children and adolescents: a systematic review and meta-analysis. Nutr Rev 76, 88-107.

21. Malik VS, Schulze MB \& Hu FB (2006) Intake of sugar-sweetened beverages and weight gain: a systematic review. Am J Clin Nutr 84, 274-288.

22. Lin P-Y, Lin F-Y, Chen T-C, et al. (2016) Relationship between sugar intake and obesity among school-age children in Kaohsiung, Taiwan. J Nutr Sci Vitaminol (Tokyo) 62, 310-316.

23. Tseng A-T, Tsao H-C, Sarukura N, et al. (2010) Development of a food composition database of refined monosaccharides and disaccharides in snacks, desserts, and beverages. Nutr Sci J 35, 146-156.

24. Shih YH, Wu HC, Pan WH, et al. (2019) Increased coffee, tea, or other sugar-sweetened beverage consumption in adolescents is associated with less satisfactory dietary quality, body fatness and serum uric acid profiles over the past 18 years in Taiwan. Asia Pac J Clin Nutr 28, 371-382.

25. Shang XW, Liu AL, Zhang Q, et al. (2012) Report on childhood obesity in China (9): sugar-sweetened beverages consumption and obesity. Biomed Environ Sci 25, 125-132.

26. Striegel-Moore RH, Thompson D, Affenito SG, et al. (2006) Correlates of beverage intake in adolescent girls: the National Heart, Lung, and Blood Institute Growth and Health Study. J Pediatr 148, 183-187.

27. Pan W-H, Kao M-D, Tzeng M-S, et al. (1999) Nutrition and Health Survey in Taiwan (NAHSIT) 1993-1996: design, contents, and operations. Nutr Sci J 24, 1-10.

28. Chen CM, Lou MF \& Gau BS (2014) Prevalence of impaired fasting glucose and analysis of related factors in Taiwanese adolescents. Pediatr Diabetes 15, 220-228.

29. Lee M-S, Pen W-H, Kai-Li L, et al. (2006) Reproducibility and validity of a Chinese food frequency questionnaire used in Taiwan. Asia Pac J Clin Nutr 15, 161-169.

30. Health Promotion Administration, Ministry of Health and Welfare (2013) The suggestion of BMI for child and adolescents. https://www. hpa.gov.tw $/$ Pages $/$ Detail.aspx?nodeid $=542 \&$ pid $=9547$ (accessed June 2013).

31. Ridley K, Ainsworth BE \& Olds TS (2008) Development of a compendium of energy expenditures for youth. Int J Behav Nutr Phys Act 5, 45. 
32. Bleich SN, Vercammen KA, Koma JW, et al. (2018) Trends in beverage consumption among children and adults, 2003-2014. Obesity (Silver Spring) 26, 432-441.

33. Stern D, Piernas C, Barquera S, et al. (2014) Caloric beverages were major sources of energy among children and adults in Mexico, 1999-2012. J Nutr 144, 949-956.

34. Terry-McElrath YM, O’Malley PM \& Johnston LD (2014) Energy drinks, soft drinks, and substance use among US secondary school students. J Addict Med 8, 6-13.

35. Rerksuppaphol L \& Rerksuppaphol S (2015) Prevalence of cigarette smoking and associated risk factors amongst middle-school students in Ongkharak District, Thailand. I Med Assoc Thai 98, Suppl. 9, S1-S8.

36. Demissie Z, Eaton DK, Lowry R, et al. (2015) The association of meal practices and other dietary correlates with dietary intake among high school students in the United States, 2010. Am J Health Promot 29, e203-e213.

37. Vereecken C, Dupuy M, Rasmussen M, et al. (2009) Breakfast consumption and its socio-demographic and lifestyle correlates in schoolchildren in 41 countries participating in the HBSC study. Int J Public Health 54, Suppl. 2, 180-190.

38. Wang M, Zhong JM, Wang H, et al. (2016) Breakfast consumption and its associations with health-related behaviors among schoolaged adolescents: a cross-sectional study in Zhejiang Province, China. Int J Environ Res Public Health 13, E761.

39. Wang W-Y (2018) The Phenomenon of Eating out for Breakfast in Taiwan and Relevant Social Changes: A Case Study of the Greater Taipei Area. PhD Thesis, National Taiwan Normal University.

40. An-Hung Fu H-CH \& Jien JJ (2013) Blood lipid profile status and factors causing abnormal blood lipid profile of senior high school students in Taiwan. J Health Sci 1, 36-56.

41. Li M, Dibley MJ, Sibbritt DW, et al. (2010) Dietary habits and overweight/obesity in adolescents in Xi'an City, China. Asia Pac J Clin Nutr 19, 76-82.

42. Perheentupa J \& Raivio K (1967) Fructose-induced hyperuricaemia. Lancet ii, 528-531.

43. Chuang S-Y, Lee S-C, Hsieh Y-T, et al. (2011) Trends in hyperuricemia and gout prevalence: Nutrition and Health Survey in Taiwan from 1993-1996 to 2005-2008. Asia Pac J Clin Nutr 20, 301-308.

44. Towiwat P \& Li ZG (2015) The association of vitamin C, alcohol, coffee, tea, milk and yogurt with uric acid and gout. Int J Rheum Dis 18, 495-501.

45. Zhang Y, Cui Y, Li XA, et al. (2017) Is tea consumption associated with the serum uric acid level, hyperuricemia or the risk of gout? A systematic review and meta-analysis. BMC Musculoskelet Disord $18,95$.

46. Song P, Yu J, Chang X, et al. (2017) Prevalence and correlates of metabolic syndrome in Chinese children: the China Health and Nutrition Survey. Nutrients 9, E79.

47. Kumar R, Khalsa DD \& Carmody JB (2016) Serum uric acid and hyperuricemia in U.S. adolescents: 40-year trends. Clin Exp Rheumatol 34, S22-S23.
48. Bobridge KS, Haines GL, Mori TA, et al. (2013) Dietary fructose in relation to blood pressure and serum uric acid in adolescent boys and girls. J Hum Hypertens 27, 217-224.

49. Luciano R, Shashaj B, Spreghini M, et al. (2017) Percentiles of serum uric acid and cardiometabolic abnormalities in obese Italian children and adolescents. Ital J Pediatr 43, 3.

50. Chen JH, Chuang SY, Chen HJ, et al. (2009) Serum uric acid level as an independent risk factor for all-cause, cardiovascular, and ischemic stroke mortality: a Chinese cohort study. Arthritis Rheum 61, 225-232.

51. Zhang W, Iso H, Murakami Y, et al. (2016) Serum uric acid and mortality form cardiovascular disease: EPOCH-JAPAN study. J Atheroscler Thromb 23, 1365-1366.

52. Rodenbach KE, Schneider MF, Furth SL, et al. (2015) Hyperuricemia and progression of CKD in children and adolescents: the Chronic Kidney Disease in Children (CKiD) cohort study. Am J Kidney Dis 66, 984-992.

53. Jha V, Garcia-Garcia G, Iseki K, et al. (2013) Chronic kidney disease: global dimension and perspectives. Lancet 382, 260-272.

54. Lin Y-C, Hsu C-Y, Kao C-C, et al. (2014) Incidence and prevalence of ESRD in Taiwan Renal Registry Data System (TWRDS): 20052012. Acta Nephrol 28, 65-68.

55. Lew Q-LJ, Jafar TH, Jin A, et al. (2018) Consumption of coffee but not of other caffeine-containing beverages reduces the risk of endstage renal disease in the Singapore Chinese Health Study. J Nutr 148, 1315-1322.

56. Rebholz CM, Grams ME, Steffen LM, et al. (2017) Diet soda consumption and risk of incident end stage renal disease. Clin I Am Soc Nephrol 12, 79-86.

57. Yuzbashian E, Asghari G, Mirmiran P, et al. (2016) Sugar-sweetened beverage consumption and risk of incident chronic kidney disease: Tehran Lipid and Glucose Study. Nephrology 21, 608-616.

58. Rebholz CM, Young BA, Katz R, et al. (2019) Patterns of beverages consumed and risk of incident kidney disease. Clin I Am Soc Nephrol 14, 49-56.

59. Lin W-T, Chan T-F, Huang H-L, et al. (2016) Fructose-rich beverage intake and central adiposity, uric acid, and pediatric insulin resistance. J Pediatr 171, 90-96.e1.

60. Nguyen S, Choi HK, Lustig RH, et al. (2009) Sugar-sweetened beverages, serum uric acid, and blood pressure in adolescents. J Pediatr 154, 807-813.

61. Chan T-F, Lin W-T, Chen Y-L, et al. (2014) Elevated serum triglyceride and retinol-binding protein 4 levels associated with fructose-sweetened beverages in adolescents. PLOS ONE 9, e82004.

62. Yi M, Wu X, Zhuang W, et al. (2019) Tea consumption and health outcomes: umbrella review of meta-analyses of observational studies in humans. Mol Nutr Food Res 63, e1900389.

63. Onakpoya I, Spencer E, Heneghan C, et al. (2014) The effect of green tea on blood pressure and lipid profile: a systematic review and meta-analysis of randomized clinical trials. Nutr Metab Cardiovasc Dis 24, 823-836. 